\title{
Nanoscale
}

A Check for updates

Cite this: Nanoscale, 2019, 11, 2938

\section{Doping of thiolate protected gold clusters through reaction with metal surfaces $\dagger$}

\author{
Rania Kazan, ${ }^{a}$ Ulrich Müller (iD b and Thomas Bürgi (iD *a
}

A new technique is introduced for doping gold nanoclusters by using a metal surface such as $\mathrm{Ag}$, Cu and $\mathrm{Cd}$ as a source of heteroatoms. The importance of the thiol ligand in the doping process is examined by following the reactions with MALDI-TOF mass spectrometry in the presence and the absence of the thiols on the surface. The doping reactions depend greatly on the type of the cluster and the availability of the ligand which is a crucial element for alloying. The thiol acts as a messenger exchanging the metal atoms between the cluster and the metal surface as revealed by the XPS studies performed on the metal surfaces.

Received 14th November 2018, Accepted 22nd January 2019

DOI: $10.1039 / c 8 n r 09214 a$

rsc.li/nanoscale indicate a collision mechanism between the reacting clusters rather than the exchange of small species between them. ${ }^{28}$ However, the detailed aspects of this mechanism remain to be explored.

Here we replace one reacting cluster by a solid surface and we show that metal exchange takes place in a cluster-surface reaction. We therefore introduce a facile new technique to dope gold nanoclusters by using a metal foil as a source of heteroatoms. We furthermore examine the importance and the role of the thiol ligand in the doping process by performing the doping reactions in the presence and the absence of the thiol ligands on the metal surface. The corresponding experiments are not possible for reactions between clusters because pure metal clusters are not stable in solution. Silver, copper and cadmium foils were employed to dope $\mathrm{Au}_{25}$ and $\mathrm{Au}_{38}$ nanoclusters, respectively. The doping reactions were followed over time by MALDI-TOF mass spectrometry. Furthermore, XPS studies were conducted on the $\mathrm{Ag}$ and $\mathrm{Cu}$ surfaces reacted with $\mathrm{Au}_{25}$ nanocluster to further understand the doping mechanism. The thiol plays the key role in the exchange of metal atoms between cluster and surface and the reaction is strongly depending on the nature of the cluster $\left(\mathrm{Au}_{25} v s . \mathrm{Au}_{38}\right)$ and the metal.

\section{Experimental}

\section{Materials and methods}

All chemicals were purchased from standard suppliers and used as received. Nanopure water $(>18 \mathrm{M} \Omega \mathrm{cm})$ was used.

The silver foil ( $99.9 \%$ pure and $0.127 \mathrm{~mm}$ thickness) was purchased from Strem Chemicals, Inc. Copper foil (Cu-ETP, 99.9\% purity and $0.5 \mathrm{~mm}$ thickness) was purchased from 
Euralliag. Cadmium foil (99.99975\% pure and $0.25 \mathrm{~mm}$ thickness) was purchased from Alfa Aesar.

Mass spectra were obtained using a Bruker Autoflex mass spectrometer equipped with a nitrogen laser at near threshold laser fluence in positive linear mode. [3-(4-tert-Butylphenyl)-2methyl-2-propenylidene] malononitrile (DCTB) was used as the matrix with a $1: 10,1: 100$ and $1: 1000$ analyte : matrix ratio. A volume of $2 \mu \mathrm{L}$ of the analyte/matrix mixture was applied to the target and air-dried.

XPS spectra were acquired on a Physical Electronics ${ }^{29}$ (PHI) Quantum 2000 Scanning ESCA Microprobe System using monochromated $\mathrm{Al} \mathrm{K \alpha}$ radiation $(h \nu=1486.7 \mathrm{eV})$ and a hemispherical capacitor electron-energy analyser equipped with a channel plate and a position-sensitive detector. The electron take-off angle was $45^{\circ}$ and the analyser was operated in the constant pass energy mode at $29.35 \mathrm{eV}$ for the spectra of the $\mathrm{Au} 4 \mathrm{f}$ peak and a step size of $0.25 \mathrm{eV}$ was used. The beam diameter is typically $150 \mu \mathrm{m}$. The binding energy is calibrated using $\mathrm{Cu} 2 \mathrm{p} 3 / 2, \mathrm{Ag} 3 \mathrm{~d} 5 / 2$ and $\mathrm{Au} 4 \mathrm{f} 7 / 2$ at $932.62 \mathrm{eV}, 368.21 \mathrm{eV}$ and $83.96 \mathrm{eV}$, respectively to within $\pm 0.1 \mathrm{eV}$ [see ISO 15472; 2010-05]. A control measurement on a pure Au foil immediately after yielded an Au $4 \mathrm{f} 7$ peak position of $84.12 \mathrm{eV}$, so a shift of $0.16 \mathrm{eV}$. To compensate for eventual surface charging, built-in electron and argon ion neutralizers were used. The base pressure of the system was below $5 \times 10^{-7} \mathrm{~Pa}$.

The spectra were analysed using the software MultiPak 8.2B. ${ }^{29}$ The atomic concentrations were calculated using the corrected relative sensitivity factors as given by the manufacturer and normalized to 100 at $\%$. The uncertainty is around $10 \%$ relative.

Ultraviolet-visible spectra of the nanoclusters were recorded on a Varian Cary 50 spectrophotometer using a quartz cuvette of $1 \mathrm{~cm}$ path length. Spectra were measured in toluene and normalized at $400 \mathrm{~nm}$.

\section{Cleaning and preparation of metal surfaces}

Before their reaction with the gold nanoclusters, the foils of $\sim 2 \mathrm{~cm}^{2}$ surface area were cut into four pieces and cleaned to remove surface contaminations. Mild cleaning procedure was applied, where silver and cadmium foils were rinsed several times by ethanol and then dried under nitrogen stream. Similarly, copper foils were first polished by diamond paste and then rinsed successively with acetone and ethanol to be later dried under nitrogen.

The thiol covered surfaces were prepared by further submerging the clean foils into a $1 \mathrm{mM}$ ethanolic solution of 2-phenylethanethiol (2-PET) for $\sim 24 \mathrm{~h}$ at room temperature to allow the deposition of the thiol monolayers on their surface. After deposition, excess thiols were removed from the surface by rinsing the sample thoroughly with ethanol and drying it under a stream of nitrogen.

\section{Reaction of gold nanoclusters with the metal surface}

First, $\mathrm{Au}_{38}(2-\mathrm{PET})_{24}$ and $\mathrm{Au}_{25}(2-\mathrm{PET})_{18}$ nanoclusters were synthesized by following the methods reported by Qian et al., and Lu et al. respectively. ${ }^{30,31}$ The synthesized clusters were charac- terized by MALDI mass spectrometry (Fig. S1†) and their UV-Vis spectra were recorded (Fig. S2 $\dagger$ ).

Then, the doping reactions were performed easily by mixing the gold nanoclusters with the corresponding metal foil in a $5 \mathrm{~mL}$ round bottom flask and adding $1.4 \mathrm{~mL}$ of toluene to completely cover the foils. The reactions were stirred with the help of the PSU-10i orbital shaker at $200 \mathrm{rpm}$ to avoid any mechanical abrasion, and samples of equivalent volumes were taken at different time intervals throughout the reaction to be dried and analysed by MALDI mass spectrometry.

The concentration of the cluster in the reaction mixture must be adapted to the surface area of the foil. For too concentrated solutions of the cluster the exchanged metal atoms are too diluted within the reacting cluster sample, leading to very low average exchange numbers. The concentrations needed were roughly estimated by comparing the number of reacting clusters to the number of surface atoms on the foil (number of adsorbed thiolates on the foil). The estimate is provided in the ESI. $\dagger$

\section{Results and discussion}

First, the $\mathrm{Ag}$ heteroatom doping of the $\mathrm{Au}_{25}$ nanocluster was investigated. The reactions were performed in the presence and the absence of the thiolate ligands on the surface of the silver foil respectively. The composition of the reaction mixtures was followed by MALDI mass spectrometry over the time span of 25 hours where samples were taken at different reaction times.

Fig. 1 shows the composition of the clusters after 2 min, $1 \mathrm{~h}, 3 \mathrm{~h}, 5 \mathrm{~h}, 8 \mathrm{~h}$ and $25 \mathrm{~h}$ of reaction respectively, in the presence of the thiolates on the silver surface. A distribution of peaks corresponding to $\mathrm{Au}_{25-x} \mathrm{Ag}_{x}(2-\mathrm{PET})_{18}$ clusters with up to four $\mathrm{Ag}$ atoms doped into $\mathrm{Au}_{25}$ was observed after only two minutes. A clear shift of the MALDI peaks towards lower masses, corresponding to higher Ag doping numbers, was detected for longer reaction times (Fig. 1a). This shows that $\mathrm{Au}_{25-x} \mathrm{Ag}_{x}(2-\mathrm{PET})_{18}$ doped nanoclusters were successfully obtained using the silver foil as a source of $\mathrm{Ag}$ heteroatoms.

Interestingly the average number of doped heteroatoms $\bar{x}$ as a function of time reveals a two phase kinetics (Fig. 1b): initially the reaction is extremely fast with a rate of 0.496 exchanged atoms per cluster and per minute, followed by a slower rate of 0.0068 exchanged atoms per cluster and per minute. The rate then further decreases with time.

$\mathrm{Au}_{25-x} \mathrm{Ag}_{x}(2-\mathrm{PET})_{18}$ alloy nanoclusters were also detected in the absence of the 2-PET ligand on the silver surface (Fig. 2). The number of the incorporated $\mathrm{Ag}$ atoms is noticed to increase with the reaction time (Fig. 2a). However, compared to the experiment where the silver foils were pre-covered with thiols (Fig. 1), the kinetics is completely different.

In the presence of adsorbed thiol on the silver foil the kinetics is initially extremely fast and slows down considerably afterwards. In the case of the neat silver foil, the reaction is 

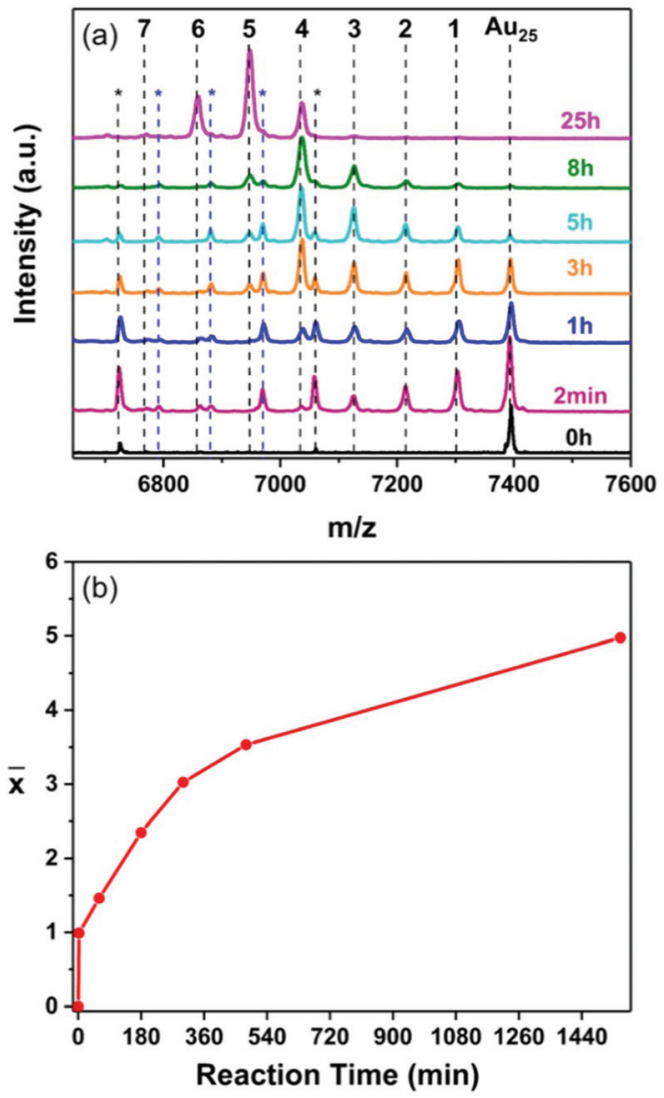

Fig. 1 (a) MALDI mass spectra of the samples taken at different times during the reaction of $\mathrm{Au}_{25}(2-\mathrm{PET})_{18}$ nanocluster with the $\mathrm{Ag}$ surface modified by 2-PET. The number of doped $\mathrm{Ag}$ atoms is marked in black above the corresponding peak where $\mathrm{M}_{\mathrm{Au} 25}=7391 \mathrm{Da}$ (Fig. S1b $\dagger$ ) and the mass difference between $\mathrm{Au}$ and $\mathrm{Ag}$ is $89.1 \mathrm{Da}$. The peaks marked with a black asterisk correspond to the loss of $A u-L$ units from the parent cluster and the ones with a blue asterisk correspond to the loss of $\mathrm{Au}-\mathrm{L}$ units from $\mathrm{Au}_{24} \mathrm{Ag}_{1}(\mathrm{SR})_{18}, \mathrm{Au}_{23} \mathrm{Ag}_{2}(\mathrm{SR})_{18}$ and $\mathrm{Au}_{22} \mathrm{Ag}_{3}(\mathrm{SR})_{18}$ respectively. (b) The average number of doped $\mathrm{Ag}$ atoms $(\bar{x})$ in the cluster, as determined by MALDI, as a function of reaction time.

very slow initially and increases its rate after about three hours only. The average number of incorporated silver atoms $\bar{x}$ in the cluster in Fig. 2b shows a sigmoidal trend with an induction period of three hours. This shows that the pre-adsorbed thiol on the foil plays an important role in this cluster-surface reaction.

It is noteworthy that the sigmoidal behaviour observed herein is usually seen in autocatalytic reactions. These reactions proceed slowly at the start (the induction period) because there is little catalyst present, before their rate increases progressively as the amount of the catalyst increases. Therefore, the doping reaction in absence of the thiol on the surface of the foil bears similarities with an autocatalytic reaction. The "catalyst" in this case could be the thiolate on the surface. Since the surface is not covered initially by 2-PET, the latter could be deposited on the surface upon the gradual decomposition of the $\mathrm{Au}_{25}$ cluster on it as will be discussed later. ${ }^{32}$ In view of the very fast reaction in the presence of the
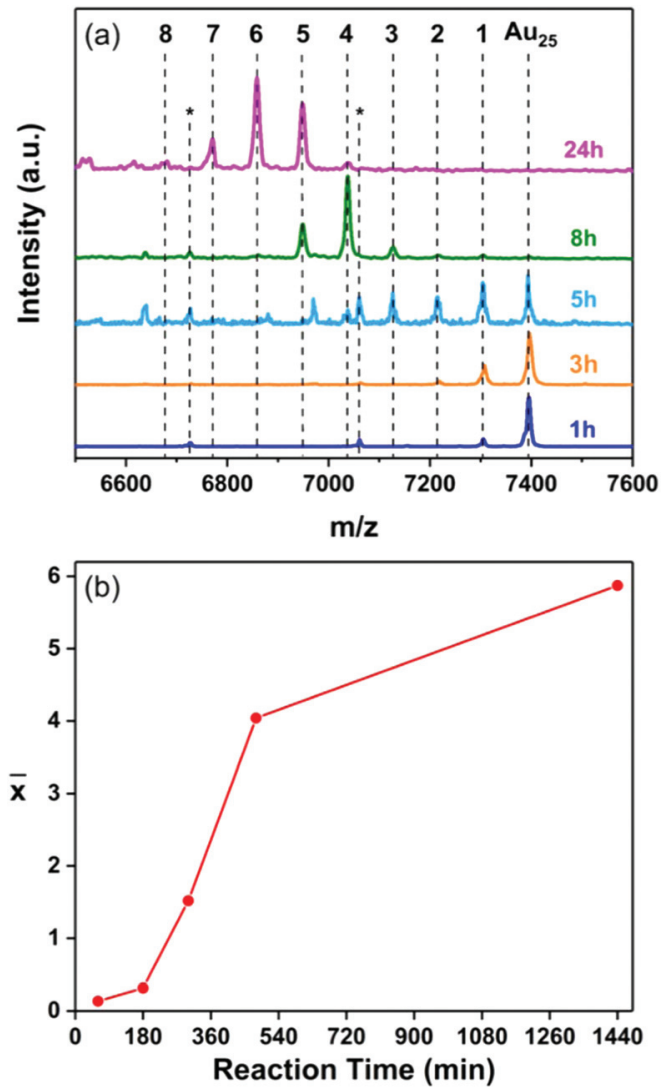

Fig. 2 (a) MALDI mass spectra of the samples taken at different times during the reaction of $\mathrm{Au}_{25}(2-\mathrm{PET})_{18}$ nanocluster with the $\mathrm{Ag}$ surface (without pre-adsorbed 2-PET). The number of doped $\mathrm{Ag}$ atoms is marked in black above the corresponding peak. The peaks marked with a black asterisk correspond to the loss of $\mathrm{Au}-\mathrm{L}$ units from the $\mathrm{Au}_{25}$ nanocluster. (b) The average number of doped $\mathrm{Ag}$ atoms $(\bar{x})$ as a function of reaction time.

initially 2-PET covered foil the decomposition rate seems comparably negligible in that case, at least at the beginning of the reaction.

The question arises why during the reaction the rate decreases by almost two orders of magnitude after short reaction time in the presence of pre-adsorbed thiol (Fig. 1). Compared to the small amount of cluster used in the experiments the silver foil represents an almost infinitely large reservoir of silver atoms. However, only the atoms at the surface are accessible for the reacting gold clusters. The reaction between cluster and surface leads to the deposition of gold on the surface (metal atom exchange, see below). This slows down the apparent reaction rate because an exchange of a gold atom from the surface with a gold atom in the cluster has no net effect. In order to test this hypothesis we estimated the amount of gold deposited on the silver surface (monolayers) by the exchange of one metal atom with the cluster $(\bar{x}=1)$ (Table S1 $\dagger$ ). This value is reached after about two minutes reaction time (Fig. 1). This estimate is easily done by relating the number of clusters in the reactor to the number of silver surface atoms of the foil. The latter number can only be esti- 
mated as the foil is polycristalline. This estimate yields a value of about 0.33 monolayers of exchanged metal atoms. After that the reaction slows down considerably. Although this is a rough estimate the value is on the same order of magnitude as a monolayer thus supporting the hypothesis that the slowing down of the reaction is related to the deposition of gold on the sliver foil. The fact that the estimate is below one full monolayer could mean that not all the surface silver atoms are exchangable. In fact, the experiment in the absence of preadsorbed thiolate shows that (initially) the surface silver atoms do not react with the cluster. Taking this together indicates that only the silver atoms attached to thiolates (possibly silver ad-atoms) are exchangable with gold atoms from the cluster. The coverage of 0.33 mentioned above could then correspond to these ad-atoms.

After the initially fast reaction of the exchangeable silver atoms the reaction continues, although at slower rate. This slower kinetics reflects replacement of gold atoms by silver at the exchangeable sites as depicted in Scheme 1.

To exclude the possibility that this cluster-surface reaction is a unique property of $\mathrm{Au}_{25}$ both reactions, with and without the adsorbed thiolate monolayer, were performed using the $\mathrm{Au}_{38}$ nanocluster. Fig. $3 \mathrm{a}$ and $4 \mathrm{a}$ show the MALDI mass spectra of both reactions taken at different reaction times. As in the case of $\mathrm{Au}_{25}$ nanocluster, $\mathrm{Au}_{38-x} \mathrm{Ag}_{x}$ alloy clusters are detected in both cases, indifferent of the availability of thiol on the metal surface.

Like for $\mathrm{Au}_{25}$ the $\mathrm{Ag}$ doping reaction of $\mathrm{Au}_{38}$ nanocluster shows a two-phase trend when the thiol is pre-adsorbed on the

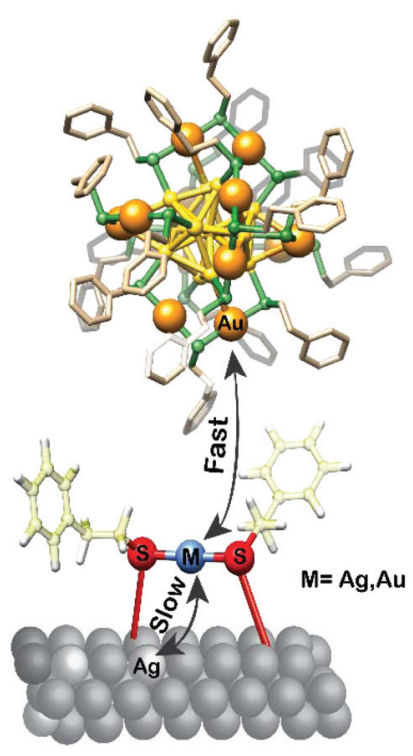

Scheme 1 Model to explain the observed two phase kinetics of the metal exchange reaction between the $\mathrm{Au}_{25}(2-\mathrm{PET})_{18}$ nancocluster and the Ag surface modified by 2-PET thiol. Some metal atoms in contact with thiolates (possibly metal ad-atoms within staples) are exchanged fast. The exchange of these atoms with surface metal atoms is slow. Color code: Yellow for $\mathrm{Au}$ in the cluster core, orange for $\mathrm{Au}$ in the staples, green for $\mathrm{S}$ and blue for $\mathrm{Ag} / \mathrm{Au}$ ad-atom on the surface.
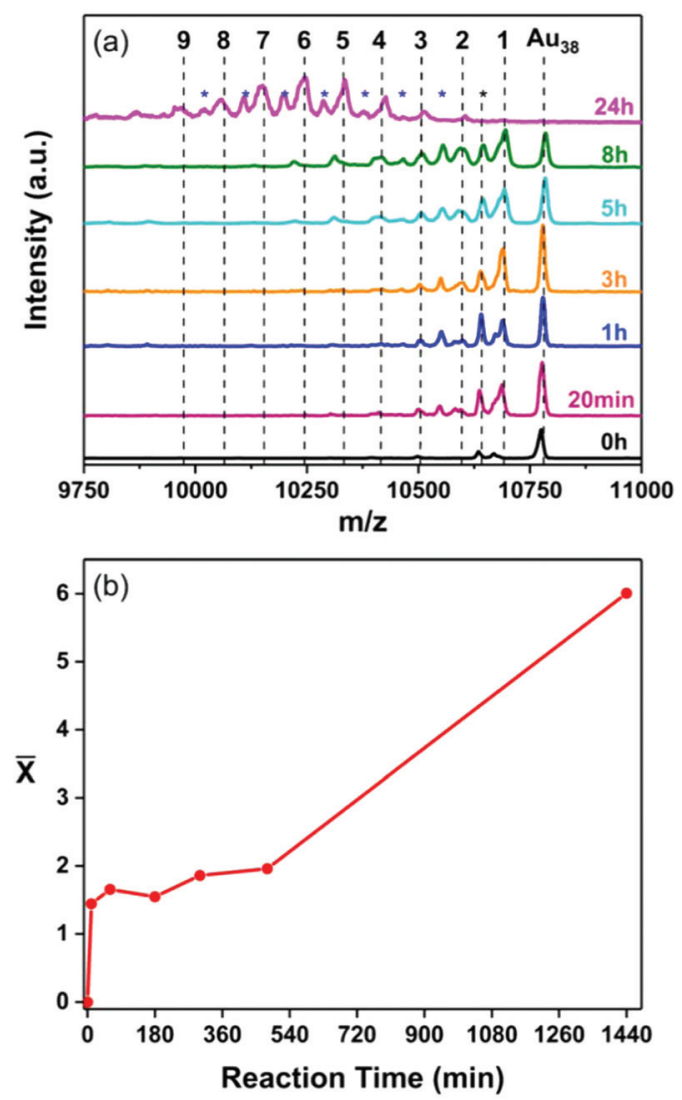

Fig. 3 (a) MALDI mass spectra of the samples taken at different times during the reaction of $\mathrm{Au}_{38}(2-\mathrm{PET})_{24}$ nanocluster with the 2-PETmodified Ag-surface where $M_{A u 38}=10778 \mathrm{Da}$ (Fig. S1a ) and $\mathrm{M}_{\mathrm{Au}}-\mathrm{M}_{\mathrm{Ag}}=$ 89.1 Da. The number of doped $\mathrm{Ag}$ atoms is marked in black above the corresponding peak. The peaks marked with a blue asterisk correspond to the loss of $L$ unit from the subsequent doped cluster. The peak with a black asterisk corresponds to $\mathrm{Au}_{38}(2-\mathrm{PET})_{23}$ species. (b) The average number of doped $\mathrm{Ag}$ atoms $(\bar{x})$ as a function of time.

surface (Fig. 3b). In the absence of the thiol the reaction is initially slow and accelerates afterwards, similar to the $\mathrm{Au}_{25}$ case discussed above (Fig. 4b).

It is worth mentioning that unlike the $\mathrm{Au}_{25}$ where the doping takes place easily, silver doping of the $\mathrm{Au}_{38}$ nanocluster in the presence of the adsorbed thiol is less effective with only two doped atoms after 8 hours (Fig. 3a). This agrees with what was previously reported in the literature for doping by direct synthesis where up to $21 \mathrm{Ag}$ atoms could be doped into $\mathrm{Au}_{25}{ }^{33}$ while only nine atoms could be introduced into the $\mathrm{Au}_{38}$ structure. $^{34}$

In the light of the successful doping of the $\mathrm{Au}_{25}$ and $\mathrm{Au}_{38}$ nanoclusters by using the silver foil as a source of heteroatoms, this technique was further employed to obtain copper and cadmium doped clusters by conducting analogous experiments with a copper and a cadmium foil, respectively.

As in the case of $\mathrm{Ag}$, the foil serves as a source of $\mathrm{Cu}$ heteroatoms. While for $\mathrm{Au}_{25}$ a monodoped cluster is observed within only 2 minutes (weak peak in the MALDI, Fig. 5a, black trace), 

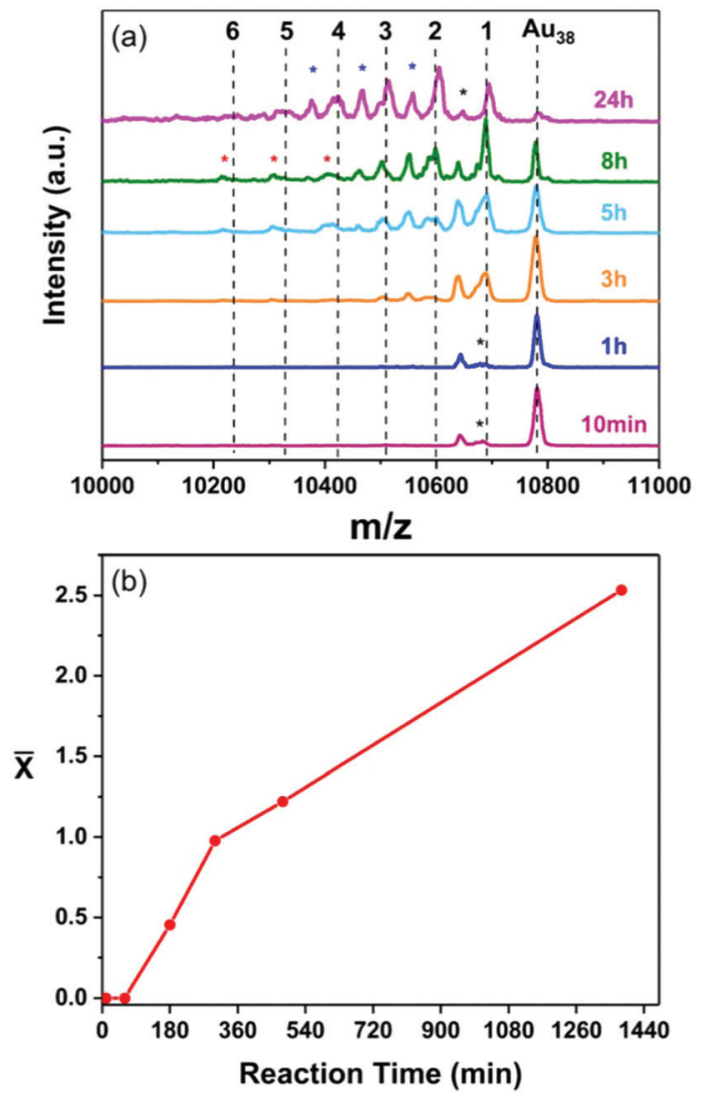

Fig. 4 (a) MALDI mass spectra of the samples taken at different times during the reaction of $\mathrm{Au}_{38}(2-\mathrm{PET})_{24}$ nanocluster with the 2-PET free Ag-surface. The number of doped $\mathrm{Ag}$ atoms is marked in black above the corresponding peak. The peaks marked with a blue asterisk correspond to the loss of 2-PET unit from the subsequent doped cluster. The peak with a black asterisk corresponds to $\mathrm{Au}_{38}(2-\mathrm{PET})_{23}$ species. The peaks marked with a red asterisk correspond to $\mathrm{Au}_{38}(2-\mathrm{PET})_{21}$, $\mathrm{Au}_{37} \mathrm{Ag}_{1}(2-\mathrm{PET})_{21} \mathrm{~S}$, and $\mathrm{Au}_{36} \mathrm{Ag}_{2}(2-\mathrm{PET})_{21} \mathrm{~S}$ respectively. (b) The average number of doped $\mathrm{Ag}$ atoms $(\bar{x})$ as a function of time.

no copper alloy cluster formed when using the $\mathrm{Au}_{38}$ cluster,

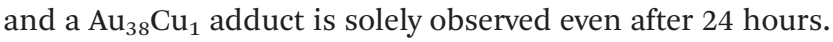

Such adduct was also reported by us recently when reacting $\mathrm{Au}_{38}$ nanocluster with $\mathrm{Cu}(\mathrm{I})$-SR complex. ${ }^{17}$ This reflects once again the dissimilarity in the doping behaviour of the two clusters.

It is noteworthy that a maximum of 15 copper atoms can be incorporated into $\mathrm{Au}_{25}$ after 1 hour (Fig. 5a). With longer reaction times the MALDI mass spectra become more and more complex due to the overlap between the $\mathrm{Au}_{25-x} \mathrm{Cu}_{x}$ peaks and the fragmentation peaks. A MALDI mass spectrum with less fragmentation (of another experiment) is provided in the ESI (Fig. S4†).

After 24 hours no peaks were observed anymore in the mass range of the $\mathrm{Au}_{25-x} \mathrm{Cu}_{x}$ cluster which we ascribe to the total decomposition of the cluster. It has been shown before that heavily copper doped clusters are unstable. ${ }^{35}$

In the same manner, $\mathrm{Au}_{25}$ nanoclusters reacted readily with the $\mathrm{Cd}$ foil covered with the 2-PET thiol and mono-doped
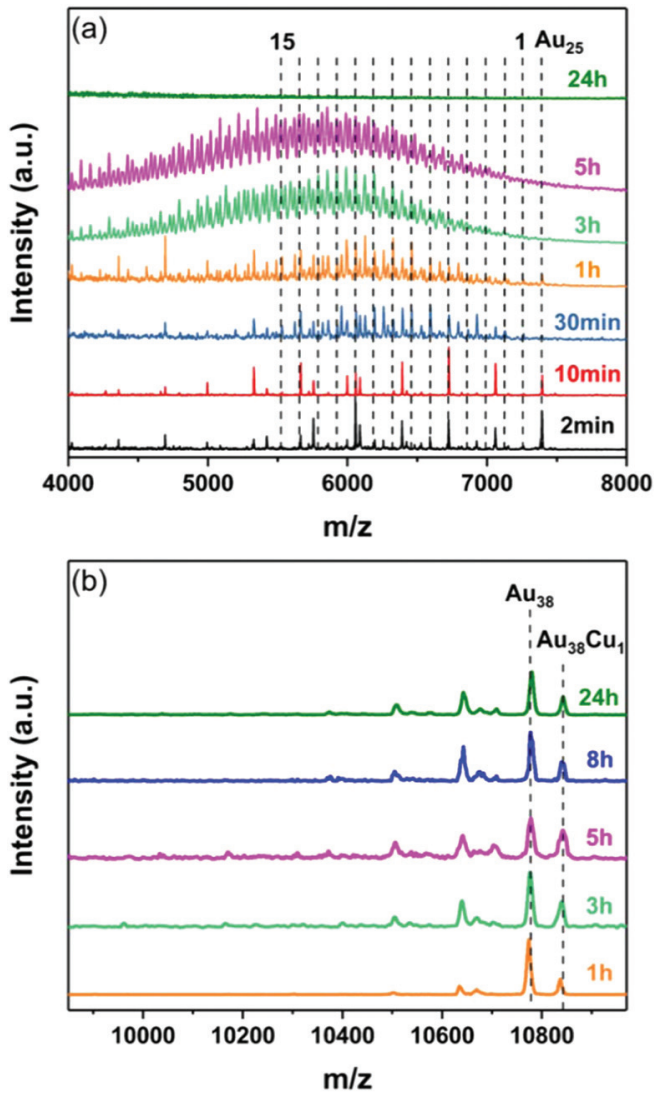

Fig. 5 (a) MALDI mass spectra of the samples taken at different times during the reaction of $\mathrm{Au}_{25}(2-\mathrm{PET})_{18}$ nanocluster with the $\mathrm{Cu}$ surface modified by 2-PET. The dashed lines mark the peaks of the different $\mathrm{Au}_{25-x} \mathrm{Cu}_{x}(2-\mathrm{PET})_{18}$ alloys where $x$ is marked in black above $(\Delta \mathrm{m} / \mathrm{z}=$ $M_{A u}-M_{C u}=133 \mathrm{Da}$ ). (b) MALDI mass spectra of the samples taken at different times during the reaction of $\mathrm{Au}_{38}(2-\mathrm{PET})_{24}$ nanocluster with the Cu surface modified by 2-PET surface (b).

$\mathrm{Cd}_{1} \mathrm{Au}_{24}$ product was exclusively detected $(\mathrm{m} / z=7309 \mathrm{Da}$, Fig. 6a). This is similar to what was reported earlier by Wang et $a l .{ }^{36}$ Nevertheless, the $\mathrm{Au}_{38}$ nanocluster remained intact and no Cd doped clusters were observed (Fig. 6b).

Interestingly, in the absence of 2-PET thiol on the $\mathrm{Cu}$ and $\mathrm{Cd}$ surfaces, neither $\mathrm{Au}_{25}$ nor $\mathrm{Au}_{38}$ alloy clusters were obtained (Fig. S5 and S6†), in contrast to the experiments with silver. A possible explanation is that on $\mathrm{Cu}$ and $\mathrm{Cd}$ foils, the gold clusters do not decompose thus avoiding the thiol deposition on their surface and therefore the doping reaction.

The experiments presented above demonstrate that this new doping method can serve as a facile and fast technique to obtain alloy clusters and provide a conclusive evidence that the thiol is a key factor in doping gold nanoclusters.

MALDI mass spectrometry provides information on the reacting cluster only. X-ray photoelectron spectroscopy (XPS) was used to study the other reaction partner, i.e. the surface.

Measurements were carried out on the thiol covered and free surfaces of silver and copper substrates that were employed in the doping reactions of the $\mathrm{Au}_{25}$ nanocluster 

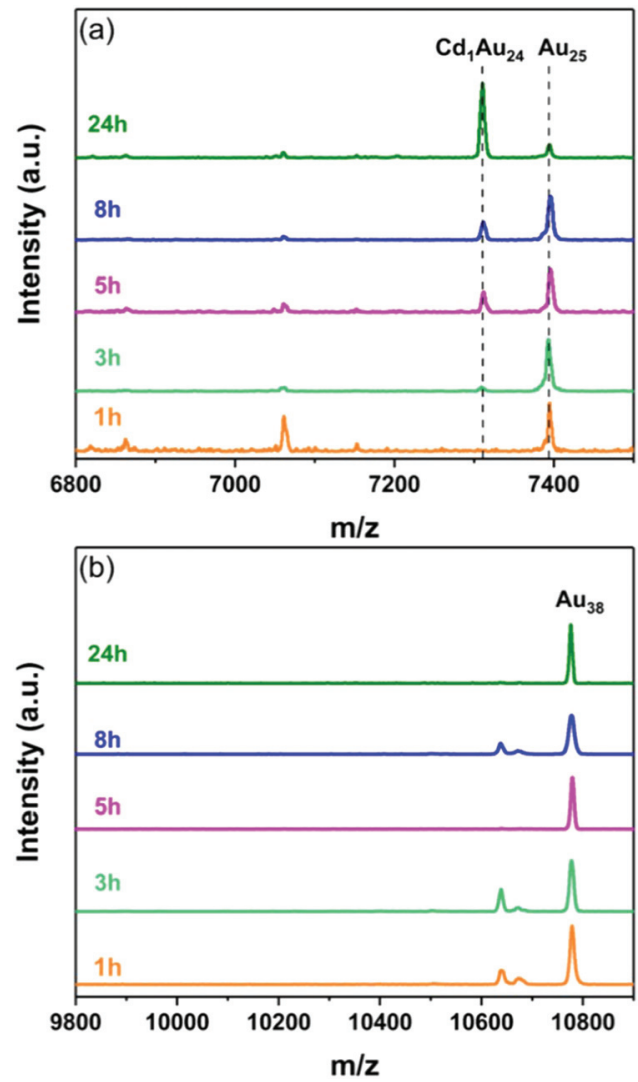

Fig. 6 MALDI mass spectra of the samples taken at different times during the reaction of $\mathrm{Au}_{25}(2-\mathrm{PET})_{18}$ (a) and $\mathrm{Au}_{38}(2-\mathrm{PET})_{24}$ nanoclusters (b) with $\mathrm{Cd}$ surface modified by 2 -PET.

respectively. This was done by removing from the reaction mixture four different pieces of the foil at certain times (two pieces in the case of thiol-free $\mathrm{Cu}$ surface). The pieces were copiously washed with toluene immediately after removal. For the same reaction, cluster solution was sampled at the corresponding times and analysed by mass spectrometry.

Fig. 7 shows the XPS spectra ( $\mathrm{Au} 4 \mathrm{f}_{7 / 2}$ and $\mathrm{Au} 4 \mathrm{f}_{5 / 2}$ ) of the reaction between $\mathrm{Au}_{25}$ and the $\mathrm{Ag}$ substrate covered with 2-PET and the spectrum of the unreacted thiol-modified foil for comparison. Unlike the reference foil, the reacted foils showed a peak corresponding to $\mathrm{Au} 4 \mathrm{f}_{7 / 2}$ at $84.25 \mathrm{eV}$ and $\mathrm{Au} 4 \mathrm{f}_{5 / 2}$ at $87.92 \mathrm{eV}$ (Fig. 7). Thus, XPS confirms the presence of gold on the silver surface, as expected for a metal exchange reaction. In agreement with the MALDI experiments (Fig. S7†) the XPS show gold on the surface already at short reaction times. The $[\mathrm{Au}] /([\mathrm{Au}]+[\mathrm{Ag}])$ ratio remains around $0.5 \%$ and for 5 hours and increases after that (Fig. S8a $\dagger$ ).

Moreover, by comparing the average number of silver atoms in the cluster (Fig. S8b†) to the fraction of gold on the silver surface $([\mathrm{Au}] /([\mathrm{Au}]+[\mathrm{Ag}])$ (Fig. S8a $\dagger$ ) as a function of time, one can see that the two are correlated. Both show a fast jump from zero to a value that remains approximately stable for five hours and an increase afterwards. This correlation is further evidence for the metal exchange between cluster and foil.

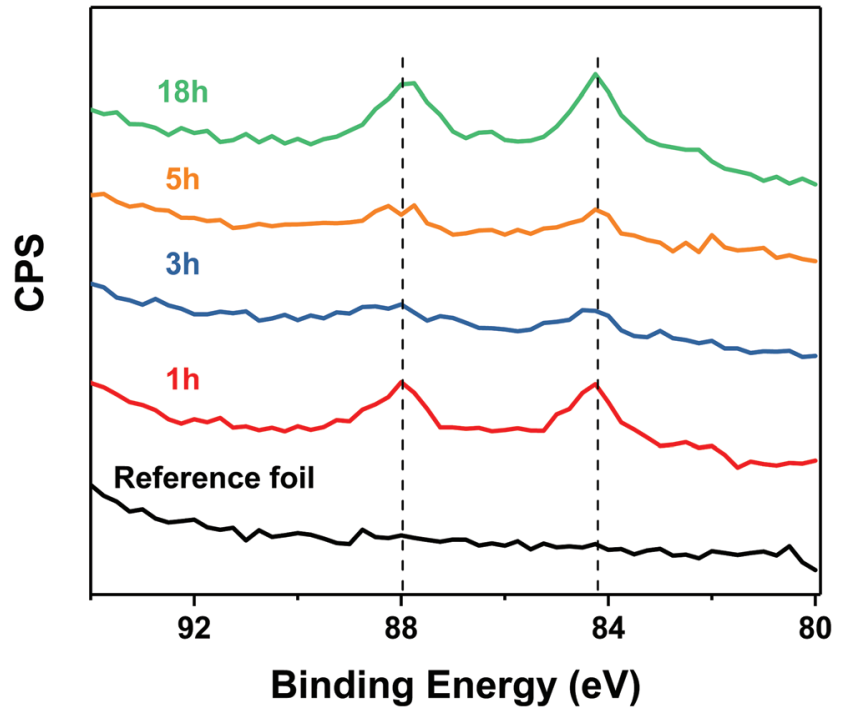

Fig. 7 XPS spectra of the silver substrates taken at different time intervals during the reaction of the $\mathrm{Au}_{25}(2-\mathrm{PET})_{18}$ nanocluster with the $\mathrm{Ag}$ foil modified by 2-PET. The black spectrum corresponds to the reference foil modified with 2-PET.

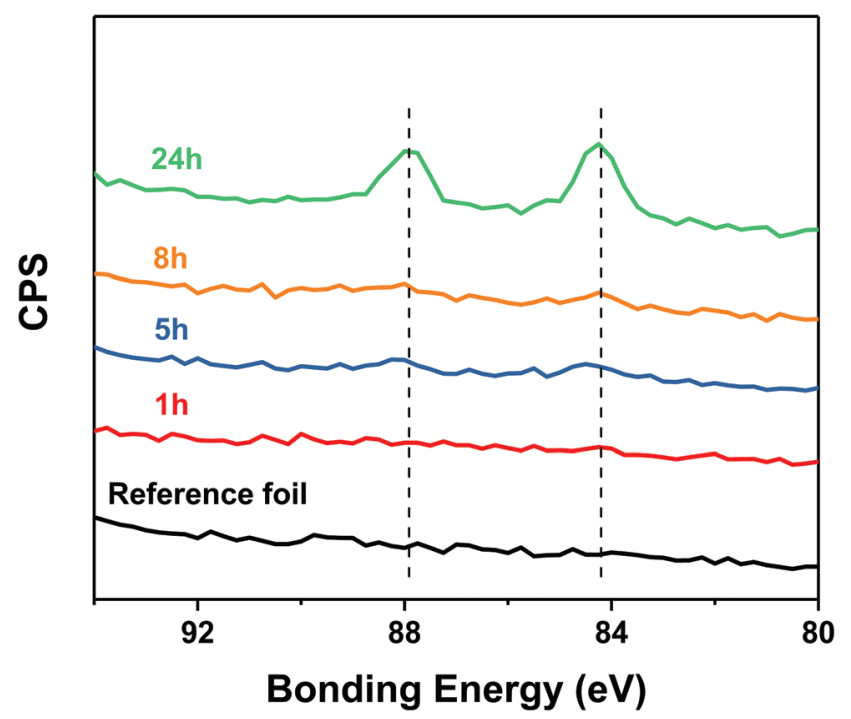

Fig. 8 XPS spectra of the silver substrates taken at different time intervals during the reaction of the $\mathrm{Au}_{25}(2-\mathrm{PET})_{18}$ nanocluster with the Ag foil (no pre-adsorbed thiol. The black spectrum corresponds to the 2-PET free reference foil.

As expected, all these samples show the presence of sulphur on the surface (Table S2 $\dagger$ ), in similar amounts as the reference sample (silver foil with pre-adsorbed 2-PET).

Likewise, the surface compositions of the thiol free silver foils reacted with $\mathrm{Au}_{25}$ were followed by XPS. As Fig. 8 shows, no gold could be detected after one hour reaction time, in contrast to the reaction with the silver foil that was pre-treated with 2-PET. However, after longer reaction time the XPS spectra show evidence for gold, in agreement with the MALDI 
experiments (Fig. S9 and S10 $\dagger$ ). In addition, sulphur could be detected (after 1 hour) on the silver foil, although the surface was initially thiol free (Table S3†).

Taken together, these observations indicate the decomposition of the $\mathrm{Au}_{25}$ cluster on the surface leading to the deposition of the thiols and the gold atoms on the foil. ${ }^{32}$

The XPS results confirm that the thiol is a key factor in the doping of the gold cluster and suggest that the latter plays the role of a "messenger" exchanging the silver and gold atoms between the cluster and the surface.

The experiments of $\mathrm{Au}_{25}$ with the copper foils reveal similar results. In the presence of the thiol ligand on the foil, $\mathrm{Au}_{25-x} \mathrm{Cu}_{x}(2-\mathrm{PET})_{18}$ clusters were detected in MALDI (Fig. S11a $\dagger$ ) and gold could be detected by XPS already at short reaction times (Fig. S12a †). These observations are completely different from the ones obtained in the absence of thiol ligand (Fig. S11b and S12b†) on the foil.

In this experiment no doping takes place according to MALDI and no gold can be detected on the $\mathrm{Cu}$ foil by XPS, at least at the beginning of the reaction.

\section{Conclusions}

A novel method to dope thiolate protected gold nanoclusters is presented where metal surfaces of silver, copper and cadmium were used to dope $\mathrm{Au}_{25}$ and $\mathrm{Au}_{38}$ nanoclusters, respectively in a cluster-surface metal exchange reaction. A strong difference in the doping behaviour of these clusters was detected depending on the nature of the metal surface used. Moreover, the role of the ligand and its importance in the doping reactions were investigated. The thiol was found to play the key role for exchanging the gold and the heterometal atoms between the cluster and the metal surface. It was found that the exchange reaction between silver foils that were pre-treated with thiols and gold clusters was initially extremely fast, followed by a much slower reaction. This result indicates that not all the surface atoms are easily exchangeable. In contrast, for thiol free surfaces the reaction is initially slow and accelerates once thiolates are deposited on the foil due to some decomposition of the clusters. This new type of cluster-surface reaction allows one to obtain surface alloys in a fast and easy manner.

\section{Conflicts of interest}

There are no conflicts to declare.

\section{Acknowledgements}

Generous support by the University of Geneva and the Swiss National Science Foundation (grant number 200020_172511) is kindly acknowledged.

\section{Notes and references}

1 I. Chakraborty and T. Pradeep, Chem. Rev., 2017, 117, 8208-8271.

2 R. Jin, C. Zeng, M. Zhou and Y. Chen, Chem. Rev., 2016, 116, 10346-10413.

3 P. D. Jadzinsky, G. Calero, C. J. Ackerson, D. A. Bushnell and R. D. Kornberg, Science, 2007, 318, 430-433.

4 R. Jin and K. Nobusada, Nano Res., 2014, 7, 285-300.

5 Y. Negishi, W. Kurashige, Y. Niihori, T. Iwasa and K. Nobusada, Phys. Chem. Chem. Phys., 2010, 12, 62196225.

6 Y. Negishi, K. Igarashi, K. Munakata, W. Ohgake and K. Nobusada, Chem. Commun., 2012, 48, 660-662.

7 S. Sharma, S. Yamazoe, T. Ono, W. Kurashige, Y. Niihori, K. Nobusada, T. Tsukuda and Y. Negishi, Dalton Trans., 2016, 45, 18064-18068.

8 H. Qian, D.-e. Jiang, G. Li, C. Gayathri, A. Das, R. R. Gil and R. Jin, J. Am. Chem. Soc., 2012, 134, 16159-16162.

9 S. Wang, Y. Song, S. Jin, X. Liu, J. Zhang, Y. Pei, X. Meng, M. Chen, P. Li and M. Zhu, J. Am. Chem. Soc., 2015, 137, 4018-4021.

10 S. Sharma, W. Kurashige, K. Nobusada and Y. Negishi, Nanoscale, 2015, 7, 10606-10612.

11 W. Kurashige, K. Munakata, K. Nobusada and Y. Negishi, Chem. Commun., 2013, 49, 5447-5449.

12 Y. Negishi, K. Munakata, W. Ohgake and K. Nobusada, J. Phys. Chem. Lett., 2012, 3, 2209-2214.

13 A. C. Dharmaratne and A. Dass, Chem. Commun., 2014, 50, 1722-1724.

14 S. Yamazoe, W. Kurashige, K. Nobusada, Y. Negishi and T. Tsukuda, J. Phys. Chem. C, 2014, 118, 25284-25290.

15 J.-P. Choi, C. A. Fields-Zinna, R. L. Stiles, R. Balasubramanian, A. D. Douglas, M. C. Crowe and R. W. Murray, J. Phys. Chem. C, 2010, 114, 15890-15896.

16 J. Chai, Y. Lv, S. Yang, Y. Song, X. Zan, Q. Li, H. Yu, M. Wu and M. Zhu, J. Phys. Chem. C, 2017, 121, 21665-21669.

17 R. Kazan, B. Zhang and T. Burgi, Dalton Trans., 2017, 46, 7708-7713.

18 C. Kumara and A. Dass, Nanoscale, 2011, 3, 3064-3067.

19 C. Kumara and A. Dass, Nanoscale, 2012, 4, 4084-4086.

20 N. Yan, L. Liao, J. Yuan, Y.-j. Lin, L.-h. Weng, J. Yang and Z. Wu, Chem. Mater., 2016, 28, 8240-8247.

21 S. Yang, S. Wang, S. Jin, S. Chen, H. Sheng and M. Zhu, Nanoscale, 2015, 7, 10005-10007.

22 C. Yao, J. Chen, M.-B. Li, L. Liu, J. Yang and Z. Wu, Nano Lett., 2015, 15, 1281-1287.

23 B. Zhang and T. Bürgi, J. Phys. Chem. C, 2016, 120, 46604666.

24 K. R. Krishnadas, A. Ghosh, A. Baksi, I. Chakraborty, G. Natarajan and T. Pradeep, J. Am. Chem. Soc., 2016, 138, 140-148.

25 K. R. Krishnadas, A. Baksi, A. Ghosh, G. Natarajan, A. Som and T. Pradeep, Acc. Chem. Res., 2017, 50, 1988-1996.

26 G. Salassa, A. Sels, F. Mancin and T. Bürgi, ACS Nano, 2017, 11, 12609-12614. 
27 B. Zhang, O. V. Safonova, S. Pollitt, G. Salassa, A. Sels, R. Kazan, Y. Wang, G. Rupprechter, N. Barrabés and T. Bürgi, Phys. Chem. Chem. Phys., 2018, 20, 5312-5318.

28 B. Zhang, G. Salassa and T. Bürgi, Chem. Commun., 2016, 52, 9205-9207.

29 Physical Electronics Inc., Chanhassen, MN 55317 (USA), https://www.phi.com/.

30 H. Qian, Y. Zhu and R. Jin, ACS Nano, 2009, 3, 3795-3803.

31 Y. Lu, Y. Jiang, X. Gao and W. Chen, Chem. Commun., 2014, 50, 8464-8467.
32 E. Pensa and T. Albrecht, J. Phys. Chem. Lett., 2018, 9, 57-62. 33 R. Jin, S. Zhao, C. Liu, M. Zhou, G. Panapitiya, Y. Xing, N. L. Rosi, J. P. Lewis and R. Jin, Nanoscale, 2017, 9, 1918319190.

34 C. Kumara and A. Dass, Nanoscale, 2012, 4, 4084-4086.

35 Y. Negishi, K. Munakata, W. Ohgake and K. Nobusada, J. Phys. Chem. Lett., 2012, 3, 2209-2214.

36 S. Wang, Y. Song, S. Jin, X. Liu, J. Zhang, Y. Pei, X. Meng, M. Chen, P. Li and M. Zhu, J. Am. Chem. Soc., 2015, 137, 4018-4021. 